\title{
INCREASING COLD-ROLLING MILL (CRM) OUTPUT BY IMPROVING FLOW THROUGH BATCH ANNEALING (BAF)*
}

Michael McDonald ${ }^{1}$ Daniel Messina² Ward Eaton ${ }^{3}$

\begin{abstract}
The cold-rolled steel output of cold-rolling mill (CRM) complexes are sometimes constrained by their batch annealing facilities (BAF). This paper examines a costeffective solution to increasing capacity and quality of the BAF thereby making additional capacity available for CRM output. The solution involves applying a structured system of scheduling and operation to the BAF, using a proven level-2 software model. The system presented has been applied to a wide variety of BAF with all the leading furnace brands. Whether using traditional $\mathrm{HN}$ atmosphere or $100 \%$ hydrogen, peak performance can be achieved when using a properly configured, on-line, physical thermodynamic model. Given that it is not practical to place a thermocouple in each coil that is being annealed, various methods have historically been used to estimate the time required to properly anneal all the coils in the charge. This paper examines how the latest technology, in wide use throughout the USA, uses a physical thermodynamic model to shorten average cycle times, thereby increasing productivity and reducing energy consumption. The paper also examines the benefit of building thermodynamically balanced charges to further optimize the BAF operation and improve quality consistency by decreasing mechanical property variations.
\end{abstract}

Keywords: BAF; Batch annealing; Bell furnaces; Bell-type; Hydrogen; HN; HNx; Level-2; Computer model; Scheduling; Stacking; Constraint; Energy savings; Green.

1 BS Metallurgical Engineering, Michigan Technological University, Houghton, MI USA and MBA Management, Wayne State University, Detroit, MI USA

2 BS Mathematics, John Carroll University, University Heights, OH USA and MS Mathematics, University of Dayton, Dayton, OH USA

3 BA Computer Science, Capital University, Bexley, OH USA and BS Systems and Controls Engineering, Case Western Reserve University, Cleveland, OH USA

* Technical contribution to the 51st Rolling Seminar - Processes, Rolled and Coated Products, October $28^{\text {th }}$ to $31^{\text {st }}, 2014$, Foz do Iguaçu, PR, Brazil. 


\section{INTRODUCTION}

Modern batch annealing facilities (BAF), whether using traditional $\mathrm{HN}$ atmosphere or $100 \%$ hydrogen, achieve peak performance when using a properly configured, online, physical thermodynamic model. Today's typical batch anneal facility produces well below its potential capacity and at higher than necessary operating costs due to inefficient cycle practices and excessive safety-margins. Given that it is not practical to place a thermocouple in each coil that is being annealed, various methods have historically been used to estimate the time required to properly anneal all the coils in the charge. This paper examines how the latest technology, in wide use throughout the USA, uses a physical thermodynamic model to shorten average cycle times, thereby increasing throughput and reducing energy consumption. The paper also examines the benefit of building thermodynamically balanced charges to further optimize the BAF operation and decrease mechanical property variation.

\section{HEATING MODEL TYPES}

Steel coils are annealed to reduce the internal strain of cold working or to improve machinability by facilitating recovery, recrystallization, and sometimes spheroidization at elevated temperatures. The time and temperature influence on these metallurgical reactions has been well documented for years, and modern batch annealing facilities (BAF) provide excellent temperature uniformity and control of atmosphere gas temperatures. If only it were practical to directly measure the temperature profile inside the tightly wound coil core during the annealing process, that temperature would be used to control the desired metallurgical reaction in each coil. This information would allow each charge of coils to be processed only as long as necessary for the metallurgical reactions to occur.

Since it is not practical to measure the core temperature of each coil, models are used to determine when the core has completed the metallurgical reactions. Models are representations of real objects or systems. Just as a model car is a representation of a real car, an anneal model represents a real anneal system. There are three basic types of models typically applied for annealing production.

\subsection{Traditional Lookup Table}

Traditional batch anneal operations break the heating portion of a cycle into two segments: heat and soak. The heat segment raises the temperature until the gas temperature reaches the required set point, and the soak segment holds at that temperature for a prescribed amount of time.

Traditional lookup tables are developed through trial and error until a certain soak time consistently produces acceptable mechanical properties. Cycles with different soak times or temperatures are developed for different quality grades. Practitioners of this approach anticipate that differences in heating rate will compensate for most of the variation in coil size and equipment performance, but also understand that additional soak time is added to compensate for unknown conditions. Typically quality issues (either caused by other problems or improper anneal) also lead to additional soak time added to the recipe. Very few modern facilities continue to use lookup tables.

\footnotetext{
* Technical contribution to the 51st Rolling Seminar - Processes, Rolled and Coated Products, October $28^{\text {th }}$ to $31^{\text {st }}, 2014$, Foz do Iguaçu, PR, Brazil.
} 


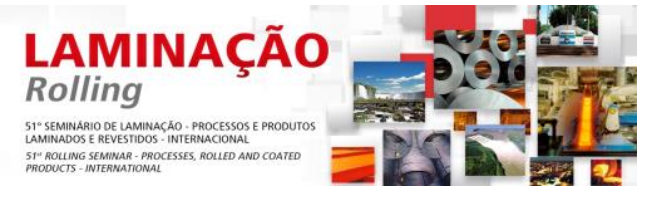

\subsubsection{Material properties}

The characterization of material properties (conductivity, specific heat, density, viscosity, etc.) under different conditions of the anneal process have also been developed for various components and constituents of the system, such as mixed atmosphere gases, carbon and stainless steel, non-ferrous materials, coatings and oils. Most of the material properties are well documented at standard temperature, but there is limited data at elevated temperatures or under annealing conditions. Development of these material properties models is fundamental to solving the heat transfer equations for the annealing process.

\subsubsection{Heat transfer properties}

Transfer of heat to and from the annealing system is described by the heat transfer properties of conduction, convection, and radiation. Conduction is the transfer of energy within a material which occurs within the various components of the annealing system. For this application, the most important equations characterize conduction across the strip in the z-direction and from lap-to-lap in the r-direction of the coil. The form of this conduction equation is as follows:

$$
c_{p} \rho \frac{\partial T}{\partial t}=\frac{1}{r} \frac{\partial}{\partial r}\left(k_{r} r \frac{\partial T}{\partial r}\right)+\frac{\partial}{\partial z}\left(k_{z} \frac{\partial T}{\partial z}\right)
$$

$T(r, z, t)$ represents the temperature of the coil at position $(r, z)$ at time, $t$. The following boundary condition is applied to each surface node: $\mathbf{q} \cdot \mathbf{n}=-k \nabla T(r, z, t) \cdot \mathbf{n}$; where vector $\mathbf{n}$ represents the outward unit normal vector of the coil and ' $k$ ' is the coil conductivity.

Annealing bases are designed with convection systems including fans, diffusers, charge plates, convector plates and inner covers to distribute the atmosphere gas and heat throughout the system. These convection systems generate both turbulent and laminar flows that are important to the heat transfer properties of the system and must be properly characterized for an accurate model.

Heat emitted from one surface to another is described by the Stefan-Boltzman law of thermal radiation modified for gray-body radiation for the annealing application $\left(\mathrm{Q}=\sigma \varepsilon \mathrm{A}\left(\mathrm{T}^{4}-\mathrm{T}_{\mathrm{c}}{ }^{4}\right)\right.$. Radiation has a big influence on heat transfer at the start of heating as well as the start of cooling. It is generally agreed that most of the annealing related surface defect (especially surface carbon, i.e. smut, and sticker breaks) happen at these two stages of the annealing process.

The boundary conditions used for solving the conduction equation consider both convection and radiation as follows:

$$
\mathbf{q} \cdot \mathbf{n}=-k \nabla T(r, z, t) \cdot \mathbf{n}=h\left(T_{s}-T\right)+\sigma F\left(T_{h}^{4}-T^{4}\right)
$$

$h$ is the convective heat transfer coefficient, $F$ is the radiation factor which considers emissivity and geometry, $T_{s}$ is the gas stream temperature and $T_{h}$ is the inner cover surface temperature.

The collection of partial differential equations that describes the annealing process is too difficult to solve analytically, so a numerical method for solving the conduction equation is applied. Extensive application of RAD-CON's annealing property models in various operations result in accurate calculations of internal and core temperatures for eavh coil in a charge.

\footnotetext{
* Technical contribution to the 51st Rolling Seminar - Processes, Rolled and Coated Products, October $28^{\text {th }}$ to $31^{\text {st }}, 2014$, Foz do Iguaçu, PR, Brazil.
} 


\section{MODEL APPLICATION}

The model's accuracy must be sustained under the various changing conditions of the production environment, such as product mix changes, new product development, equipment wear, and process events. Only physical models can handle these types of changes and remain accurate without significant redevelopment. Physical models are applied to the anneal process as either static models run prior to the process, or dynamic models that use process feedback as additional input.

\subsection{Static/Off-Line}

A static model works like a set-up model. Variables for the charge such as coil configuration, coil dimensions, core temperature requirement, convector plate types, and temperature and atmosphere strategy are used to calculate a heating time. The charge is then annealed to the calculated heating time unless an operator intervenes to modify the heating time.

\subsection{Static/Off-Line With Automatic Adjustment}

An improvement on a strictly static application of a physical model would be to modify the required heating time based on process feedback or events. Some applications use the output of the off-line calculation to include a gas stream profile for the complete process. If the actual feedback temperature deviates from this projected profile by a significant amount, heating time is added. This is typically implemented but halting a count-down timer.

\subsection{Dynamic/On-Line}

RAD-CON's application of the physical model also starts with an off-line calculation for planning followed by actual process feedback used to recalculate heating requirements during the process. Process feedback depends on the installation, but can include values for furnace and gas stream temperature; hydrogen, nitrogen or other atmosphere flow; fan current and frequency; and ambient air and water temperature. This dynamic feedback and calculation allows for the most accurate coil temperature calculations for optimal cycle times.

\section{MODEL ACCURACY}

The objective of an anneal model is to determine the minimum anneal time necessary to meet metallurgical requirements without the waste of over annealing. The true benefit to operating cost, production capacity, and quality is found with high levels of accuracy in the production environment.

* Technical contribution to the 51st Rolling Seminar - Processes, Rolled and Coated Products, October $28^{\text {th }}$ to $31^{\text {st }}, 2014$, Foz do Iguaçu, PR, Brazil. 


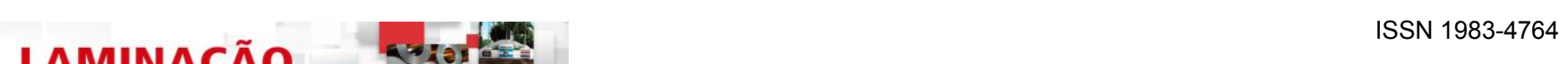

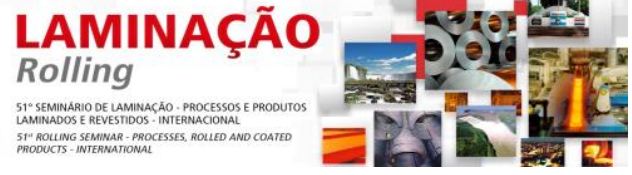

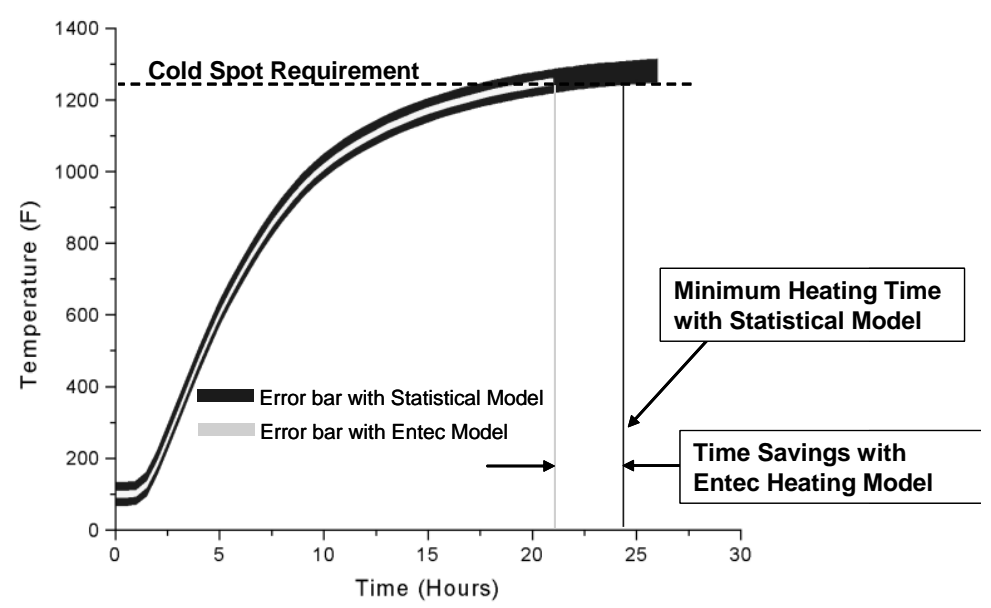

Figure 1: Impact of Calculation Error

Figure 1 compares the typical error of a very good statistical model with RAD-CON's on-line physical model. The calculation error is shown for a coil's cold spot temperature calculation with each model. To avoid under annealing, each model must exceed the cold spot temperature requirement with enough buffer to compensate for the model error. On average, the reduced error of the physical model requires shorter heating times. Typical savings are $10 \%$ to $15 \%$ of heating time, depending on the existing model other shop conditions.

The reduction in heating time will decrease the amount of fuel, electricity, and atmosphere gas consumed, lowering operating costs. Typical North American installations are saving $\$ 0.25 /$ ton for $\mathrm{H}_{2}$ bases and over $\$ 1.10$ /ton for $\mathrm{HN}$ bases. Throughput capacity is also increased since furnace and base time are reduced. In many cases, adding the RAD-CON model has reduced the amount of capital equipment necessary to meet production requirements for a new or expanding facility.

Charge-to-charge reduction in mechanical property variation is found due to decreased heating time buffers. This benefit is further improved for the coils within a charge through the use of the Stacking Model.

\section{ADVANCED APPLICATION OF MODELS}

RAD-CON has implemented its physical model to determine heating and cooling times since the late-1970s. In late-1990s, improvements in computing power have allowed for a tight mesh to be applied to the coordinate system and time intervals of RAD-CON's physical model while still using standard computer processors. This capability and a strong motivation to improve performance in customer operations led to the development of RAD-CON's Stacking Model, Cleanliness Model and Sticker Break Model.

\subsection{Stacking Model}

Many factors must be considered when configuring an anneal charge. Certain coils may be "rushed" to make customer delivery; some coils can only go on certain bases; while other coils can not be annealed in the same charge; all with the objective to build a charge as tall as possible. With these restrictions, it is almost impossible for a human to also consider the heating time for each coil and make them as close as possible.

* Technical contribution to the 51st Rolling Seminar - Processes, Rolled and Coated Products, October $28^{\text {th }}$ to $31^{\text {st }}, 2014$, Foz do Iguaçu, PR, Brazil. 


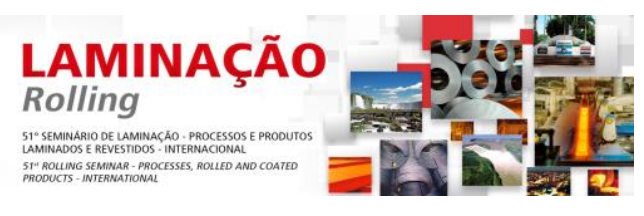

The RAD-CON Stacking Model uses linear programming methods to consider all of the constraints of charge building. Many more constraints can be considered by the computer than a human could possibly consider. Most importantly, the Stacking Model uses a simplified version of the physical model to determine the relative heating time of each coil in each potential stack position. Controlling the relative heating time required for each coil develops thermodynamically balanced changes. This decreases mechanical property variation, reduces the average heating times, and increases throughput.

\subsection{Cleanliness Model}

Surface carbon and soot are not acceptable for most critical sheet applications. Most facilities have found that surface carbon levels can be controlled by slowing down the heating rate while the rolling oils volatilize from the surface. This solution adds operating cost and reduces throughput capacity. An alternative is to control the internal coil temperatures, where the oil is volatilizing, as well as the atmosphere flow. This solution provides the same quality with a reduced impact on cost and throughput.

Requiring physical model accuracy at low temperature, during the time rolling oils volatilize, poses a technical challenge. In these early stages of the annealing process, the rolling oil modifies the atmosphere gas, changing conduction. The large temperature gradients in the system can magnify any poor assumption or error in the model. Successfully meeting these challenges allowed RAD-CON to model the process in order to modify heating rates and atmosphere flows at precise settings to meet quality requirements in the most economical manner.

\subsection{Sticker Break Model}

Annealing related sticker breaks are typically caused by the compressive forces generated as the outside laps of a coils contract during cooling. Most facilities have found that they can prevent this defect by controlling the temperature gradient within, coils (and therefore the compressive force). A typical control method is to leave the furnace on the base to perform a furnace cool for a period of time. This solution reduces shop capacity and increases mechanical property variation. RAD-CON has a more productive solution.

RAD-CON uses the physical model to predict the temperature gradient during potential future events, such as furnace removal, spray cooling, etc. The desision to execute or delay events is made is made by the model. This solution manages the seasonal impact of sticker breaks with ambient air and water temperature, and allows the facility to operate at peak capacity in most situations.

\section{CONCLUSION}

Because there is presently no practical means to measure the inside of strip coils being batch annealed on a daily production basis, models are used. The more accurate the model, the more efficient the operation. In general, the Mathematical Physical Model is the most accurate model type. The specific performance and flexibility of a Mathematical Physical Model depends on which physical factors have been considered and the various methods used in the model's design. In other

* Technical contribution to the 51st Rolling Seminar - Processes, Rolled and Coated Products, October $28^{\text {th }}$ to $31^{\text {st }}, 2014$, Foz do Iguaçu, PR, Brazil. 
words, all physical models are not alike. On-line models adjust to the dynamic conditions measured during the annealing progress. Off-line models are used to build stacks with the goal that all coils will complete annealing simultaneously. Ultimately, the true test of a model is its ability to accurately and repeatedly predict the temperature profile inside the coils at the critical points in the annealing cycle that affect mechanical properties, cleanliness, and sticking.

The accuracy and flexibility of the RAD-CON model has led to improved performance at BAF throughout the USA. Using its accurate temperature profile, RAD-CON has developed additional capabilities to assist the BAF: intelligent stacking, cleanliness through controlled oil volatization, and sticker avoidance through controlled temperature differential. Customers typically receive more than $10 \%$ additional throughput and a $6-10 \%$ reduction in utilty cost while improving product quality consistency.

* Technical contribution to the 51st Rolling Seminar - Processes, Rolled and Coated Products, October $28^{\text {th }}$ to $31^{\text {st }}, 2014$, Foz do Iguaçu, PR, Brazil. 